\title{
Automatic Engine Start-Stop and Automatic Headlight Dimming Modular System
}

\author{
Ajay Sreekumar \\ UG Scholar \\ Dept of Electrical and Electronics Engineering, \\ Mar Baselios College of Engineering and Technology, \\ Trivandrum, India \\ Shinoj Philip John \\ UG Scholar \\ Dept of Electrical and Electronics Engineering, \\ Mar Baselios College of Engineering and Technology, \\ Trivandrum, India
}

\author{
Jonathan Joseph Cleetus \\ UG Scholar \\ Dept of Electrical and Electronics Engineering, \\ Mar Baselios College of Engineering and Technology, \\ Trivandrum,India \\ Sajeevan R S \\ UG Scholar \\ Dept of Electrical and Electronics Engineering, \\ Mar Baselios College of Engineering and Technology, \\ Trivandrum, India
}

\author{
Dr. Nisha G K \\ Professor \\ Dept of Electrical and Electronics Engineering, \\ Mar Baselios College of Engineering and Technology, \\ Trivandrum, India
}

\begin{abstract}
The national mandate is being set forth by the Environmental Protection Agency or EPA to increase fuel efficiency of vehicles and thereby reducing emissions of greenhouse gases (GHG) by $5 \%$ each year for all new vehicles of various categories such as light heavy duty trucks, medium duty cars and mid-size cars. Usage of an automated start/stop (SS) technology in a passenger vehicle is an effective way to increase fuel efficiency in a cost effective way and it will also reduce emissions. In urban areas a vehicle spends most of its time in idle condition, this may be due to various reasons, one of the main reason is due to heavy traffic and this increases fuel consumption as well as air pollution. In such scenarios we can shut down the engine to save fuel and this will reduce pollution and the engine is restarted as the driver demands torque for acceleration. Most of the drivers use high beam light while driving at night and this can cause discomfort to people travelling in the opposite direction. This can even cause partial blindness to people on the opposite side. With the stop/start system activated the engine is automatically turned off and goes to standby mode when the vehicle is brought to a stop at traffic lights, stop signs and in traffic jams. The system uses sensors to monitor the vehicle motion, power requirement etc. With an ultrasonic sensor the vehicle distance and speed is calculated and by using a photo sensor or camera whether head light or tail light of a car can be distinguished.
\end{abstract}

\section{INTRODUCTION}

Conservation of energy and Global Warming are one of the major issues faced by mankind. Effluents from the motor vehicles are one of the highest contributors for rise in Carbon-Di-Oxide levels. Pollution from vehicles can be classified into mainly two types: primary and secondary. The former is when the pollutants are directly emitted to the atmosphere; and the latter results from the chemical reactions that occur between atmosphere and pollutants. The other thing that we came across is one of the major problems faced by the drivers during night time driving; the momentary blindness caused due to the high beam light of the incoming motor vehicle. This momentary blindness causes the driver from seeing the road and surrounding clearly, and thus it may lead to accidents. The number of crashes happening at night is way higher than that occurring during the day. This paper aims to present a solution for the problems faced during idling and the ones during the night time driving.

For reducing the emissions and fuel wastage due to idling at traffic, we are introducing a system - Automatic Engine Start/Stop, which turns off or restarts the IC engine thus reducing the time the engine keeps idling when stopped in traffic signals, thus it ensures reduction in emissions and fuel consumption. The gain from implementing this system is around the range of 3 to $10 \%$, and has a potential of around $12 \%$. The start stop system stops the engine when the car is completely in a stationary position while at traffic signals. Data is collected about the vehicle's motion and is then sent to the microprocessor for processing, the microprocessor processes the obtained data and sends the required control action whether or not to stop the vehicle. When the vehicle is put into a gear by the driver, the IC engine is restarted.

Manual dipping of the headlight in a motor vehicle during night time driving is a tiresome process. This burden on the driver can be reduced by introducing an automatic dimming headlight system. This system ensures that the driver has one less thing to worry about during night time driving. When an oncoming vehicle's light falls on the LDR which is placed in the front side of the vehicle, it sends a signal to the microcontroller. The microcontroller verifies the data received and sends a control action which then dims the headlight. When the upcoming vehicle 
passes, the light falling on the LDR stops and the headlight is then turned on to a high beam.

\section{PROPOSED SYSTEM}

Our project is a modular system which consists of two main modules which are combined into a single compact system. There are two parts in the block diagram. The first part of the block diagram is the headlight module while the second part is the engine shutoff module. The headlight module consists of the light dependent resistor (LDR), driver circuit and the headlight. The light dependent resistor gives the input signal to the driver circuit after detecting light of certain intensity. The driver circuit consists of a transistor and a relay which controls the headlight and thus dims the headlight if it is on bright and it returns the dimmed headlight to bright after the vehicle from the opposite lane has passed our vehicle. The engine shutoff module consists of vehicle sensors, microcontroller, buzzer, driver circuit and the engine. The vehicle sensors mainly the odometer and the neutral gear sensor give the signals to the microcontroller. The microcontroller then analyses the signals and then takes a logical route and gives an input signal to the driver circuit. The driver circuit consists of a transistor and relay which performs two activities that is it activates a buzzer thus making the user aware that the engine is going to shut off and it also shuts off the engine after the timer runs out. The whole system is compact and can be stored inside the glove box of the vehicle.
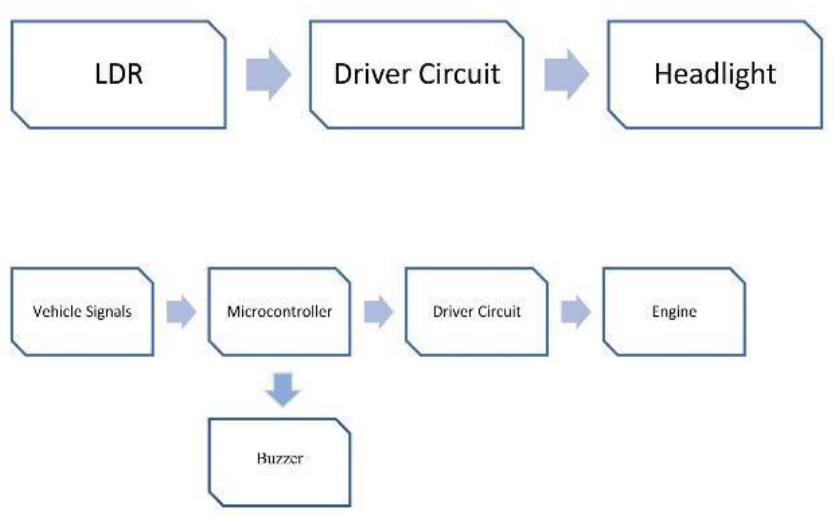

Figure 1- Block diagram

\section{COMPONENTS DETAILS}

\section{1) $\mathrm{ESP} 32$}

ESP32 is a low cost System on Chip (SoC) microcontroller and has Bluetooth and Wi-Fi modules thus making it very ideal for embedded systems and Internet of Things (IoT) projects. It can work in a wide range of operating temperature that is between $-400 \mathrm{C}$ to $125 \mathrm{OC}$.It is adaptable to any changes in the external environment and can remove any external circuit imperfections. Its power consumption is very low and it uses many types of software to achieve this. It also has several features like dynamic power scaling, various power modes and fine- grained clock gating. It also has filters, in-built antenna switches, power management modules, power amplifier, low-noise receive amplifier, and low-noise receive.. It also supports lwIP protocol and Freertos.

\section{2) $\mathrm{BC} 547$}

It is a NPN transistor with a DC current gain of maximum 800 . It has a collector current of $100 \mathrm{~mA}$ and emitter base voltage of $6 \mathrm{~V}$. It has a maximum base current of $5 \mathrm{~mA}$. In the BC547 when the base pin is grounded, the collector and emitter will be open (reverse bias) and when the base pin receives a signal then the collector and emitter will be closed (forward bias). Here the gain of 800 is used to imply the transistor's amplification capability. The transistor reaches the saturation region when the current flowing through the collector and emitter is $100 \mathrm{~mA}$. In this region a maximum voltage of $200 \mathrm{mV}$ and $900 \mathrm{mV}$ across the collector-emitter (VCE) and base-emitter (VBE) respectively is permissible. The cut off region is the region where the base current is zero, here the base emitter voltage is nearly $660 \mathrm{mV}$.

\section{3) Buzzer}

A buzzer is an audio device which provides an auditory signal to create awareness to the user about a certain parameter. It can be piezoelectric, mechanical or electromechanical.

\section{4) Diode 1 N4001}

$1 \mathrm{~N} 4001$ is a power diode and has a higher current rating which also means a higher forward voltage. It has a current rating of $1 \mathrm{~A}$ and forward voltage rating of $1.1 \mathrm{~V}$. In our project the diode is used in our power supply circuit as it helps to provide a unidirectional current to the 7805 .

\section{5) Relay}

It acts as an electromechanical switch and acts as a bridge between a small current using circuit and a high current using circuit and helps to switch on the larger circuit by the smaller current using circuit. A $12 \mathrm{~V}$ normally closed relay is used to dim the headlight when a vehicle is detected in the opposite direction by the LDR and it is also used in the engine shutoff module and helps to switch off the engine when a signal is received from the microcontroller.

\section{6) Light Dependent Resistor}

LDR helps to detect the various intensities of light and accordingly its resistance changes with the changes in the light intensities. The resistance of the LDR is high when it is dark and its resistance decreases with the increase in the intensity of the light. The sensitivity of the LDR depends on the wavelength of the incident light on the LDR. LDR is used to detect an approaching vehicle in the opposite direction as the light from the headlight will be detected and thus the resistance will be decreased and this allows a current to flow through the transistor and hence the relay will be activated and the headlight will be dimmed. 


\section{WORKING}

The vehicle module consists of three main parts which are:

\section{i. Power supply circuit}

ii. Headlight auto dimming module

iii. Engine auto shutoff module.

The power supply circuit provides power to both the headlight dimming module and the microcontroller which is part of the engine shutoff module. All of these three parts are combined together to form a compact modular system which can be attached to any existing or new vehicles.

\section{1) Power Supply Circuit}

The circuit consists of the battery, diode, capacitors, IC7805 and LED. The $12 \mathrm{~V}$ battery of the vehicle is the input given to the power supply circuit. This input then passes through the diode D1 which provides a unidirectional current and gives very high resistance in the reverse direction. The capacitor $\mathrm{C} 1$ gives the necessary filtering and then the filtered signal is given to the IC7805. The IC7805 is responsible for the voltage conversion. It converts the $12 \mathrm{~V}$ input signal to a $5 \mathrm{~V}$ output signal. The input signal is given to legs 1 and 2 and the output signal is taken from legs 2 and 3, where leg 2 is grounded. Here the voltage difference between the input and output is released in the form of heat. The $5 \mathrm{~V}$ output of the IC7805 is then passed through the capacitor $\mathrm{C} 2$ which provides filtering and also provides transient response. A LED is present which gives the user a visual feedback that the power supply circuit is working. This $5 \mathrm{~V}$ output produced by the power supply circuit is used by both the headlight dimming module and the microcontroller which is part of the engine shutoff module.

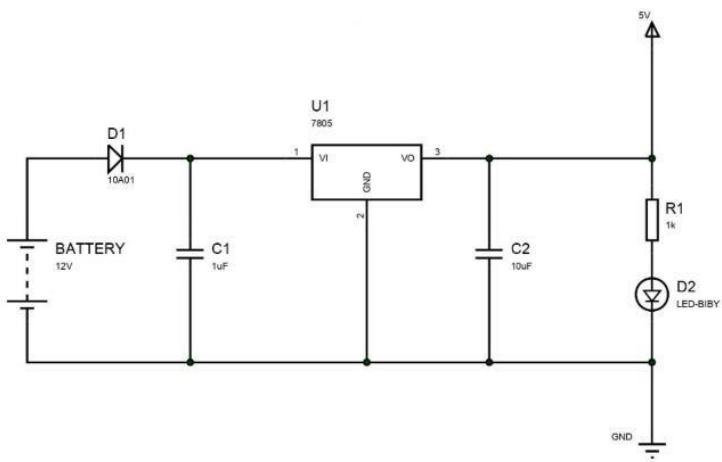

Figure 2- Power Supply Circuit

\section{2) Headlight Auto Dimming Module}

The module consists of a light dependent resistor (LDR), transistor, relay, resistors and the headlight. This module receives an input power of $5 \mathrm{~V}$ from the power supply circuit. The resistance of the light dependent resistor changes with the intensity of light that is incident on it.

The resistance decreases with the intensity of the light incident on it. So when a vehicle comes from the opposite lane, the light from its headlight will be incident on the
LDR of our vehicle. So when the intensity of light reaches a certain threshold, a signal is generated which is given as the input to the base of the transistor Q2 through a resistor R3 which helps to protect the transistor Q2 by limiting the base current to $5 \mathrm{~mA}$. When the transistor receives the base signal, it is forward biased and thus the transistor is closed and hence an output signal is given to the $5 \mathrm{~V}$ relay. The relay then magnetises the electromagnet and thus opens the closed terminals and thus dims the headlight. When the vehicle in the opposite lane passes by the light incident on the LDR decreases and thus its resistance increases and the signal to base is removed. This causes the transistor to be switched off and hence the relay will be moved to its original position and hence the headlight will be in the bright mode.

This module facilitates safe passage for both the vehicles without blinding the other and helps to automate the bright function of the headlight by switching between the bright and dim mode of the headlight according to the oncoming traffic in the opposite lane.

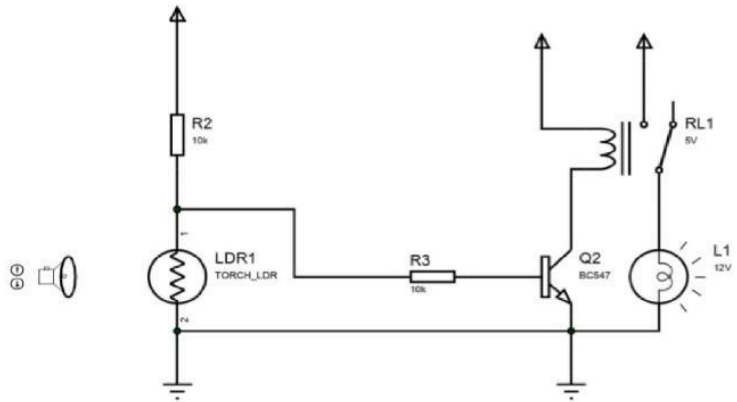

Figure 3- Circuit diagram Headlight Auto Dimming Module

\section{3) Engine Auto Shut Off Module}

This module consists of the vehicle sensors like the odometer and neutral gear sensor, microcontroller, buzzer, transistors, relays, LED's and the engine. Here the microcontroller receives the input power from the power supply circuit. The $5 \mathrm{~V}$ input voltage is converted to $3.3 \mathrm{~V}$ using a voltage divider. The microcontroller is the brain of the module; it receives input signals and analyses it logically before giving an output. The input signals are provided by the odometer and neutral gear sensors through the pins GPIO32 and GPIO33 respectively. The odometer sensor sends a signal to the microcontroller when the vehicle is not moving and the neutral gear sensor gives a signal when the vehicle is in neutral. Both of these signals are used so as to make the system fool-proof and complements the existing system thus making it an added asset and a hassle free experience for the user. The microcontroller analyses both the signals and takes a decision to whether to shut off the engine or not. This logic used by the microcontroller for starting and stopping the engine is explained using the table which is given below: 
Table 1- Engine Auto Shut Off Explanation

\begin{tabular}{|c|c|c|}
\hline Odometer & Neutral Gear & Engine \\
\hline R & G & ON \\
\hline R & N & ON \\
\hline NR & N & OFF \\
\hline
\end{tabular}

Note: NR-Not Running, R-Running, N-Neutral, G-Gear

If the odometer and neutral gear sensor gives a signal that is running and in gear then the engine is left running and not shut off, as the vehicle is in motion. If the odometer and neutral gear sensor gives a signal that is running and in neutral then the engine is left running as the vehicle is in down hill motion and a need for engine power should not be disrupted for effective engine braking as required by the user. The engine is only shut off if both the odometer and neutral gear sensors give a signal that is not running and is in neutral respectively, which means the vehicle is at rest.

So after microcontroller analyses and makes a logical decision to shut off the engine after the given parameters are met as explained above, it starts a timer of 3 seconds before shutting down the engine and it also gives an output signal to the buzzer circuit through the GPIO19 pin. Both the timer and buzzer feedback is given so that the user can make a decision to allow the shutting off of the engine or can abort it by changing the gear from neutral. The output signal is given to the buzzer circuit as soon as the timer starts so that the user has enough time to decide and so as to prevent an abrupt shutoff of the engine. The output signal from the microcontroller is given to the base of the transistor Q1 and thus the transistor is forward biased and the circuit is complete. Hence the buzzer is activated and a warning alarm is given to the user to notify about the engine shutoff. After the 3 seconds timer is done then an output signal from the microcontroller is given to the base of the transistor Q3 through the GPIO5 pin and thus the transistor is forward biased and an output signal is given to the $5 \mathrm{~V}$ relay. This is an always closed relay and thus when the signal from the transistor is given as input to the relay it magnetizes the electromagnet and hence the two closed terminals are opened. Thus the spark plugs of the combustion system will be deactivated and the engine will be shut off. So when the user changes the vehicle to gear from neutral, a signal is received from the neutral gear sensor and thus the signal to the base of the transistor Q3 is removed and thus the electromagnet of the relay will be demagnetized and the terminals will be closed. There are additional LEDs which are connected through GPIO17 and GPIO16 pins, which are used to notify the user if the engine shutoff system is working properly or not. This causes the spark plugs to be activated and hence combustion is resumed and the engine starts again. This system thus helps in increasing fuel efficiency and engine life, while decreasing the emissions and pollution caused by the vehicle.

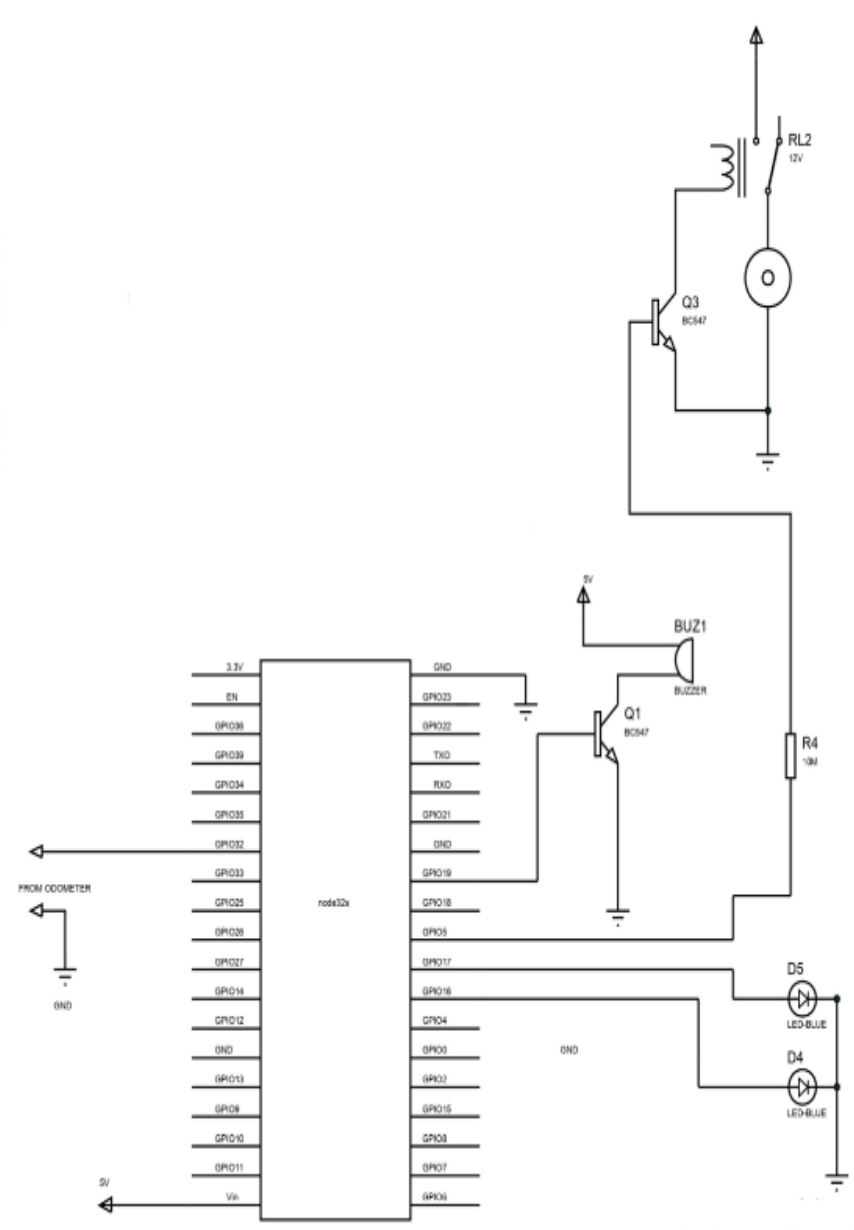

Figure 4- Circuit diagram Engine Auto Shut Off Module.

\section{SIMULATION RESULTS}

The simulation of the various circuits in the project is done in Proteus 8 Professional software.

\section{1) Simulation of Power circuit}

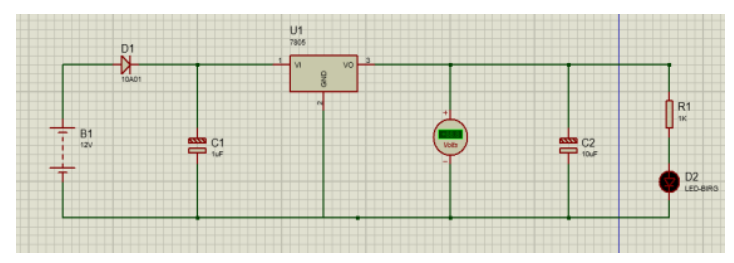

Figure 5- Proteus Simulation of power circuit.

Above figure shows the simulation of the power circuit. It consists of a battery of $12 \mathrm{~V}$ which is converted into $5 \mathrm{~V}$ using a voltage regulator IC U1- 7805. Here a diode D1 is used to provide a unidirectional current to the regulator. Here the output is verified using a LED which glows when a $5 \mathrm{~V}$ supply is given to one of its terminals. Also, the output of the regulator is confirmed to be $5 \mathrm{~V}$ by using a voltmeter as shown in the circuit. 


\section{2) Simulation of Headlight Controller}

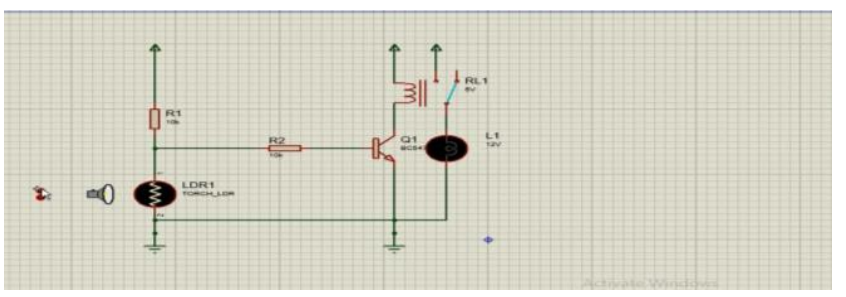

Figure 6- Proteus Simulation of Headlight Controller

Figure 6 shows the proteus simulation diagram of the headlight controller. Here the demonstration is done with the help of a light source. When the light is far away from the LDR, the transistor does not turn ON and the headlight represented by LED L1 is connected to the supply terminal of the relay and it glows bright. When the light source moves near the LDR the transistor turns ON which activates the relay. Thereby then the relay is switched to OFF mode and the headlight L1 dims. Here the output is verified by the LED.

\section{HARDWARE RESULTS}

The hardware part of the project is explained in different sections.

\section{1) Power Circuit}

In this project a simple power circuit is used in which $12 \mathrm{~V}$ source is converted into a $5 \mathrm{~V}$ supply. The hardware circuit of the power supply is as shown below.

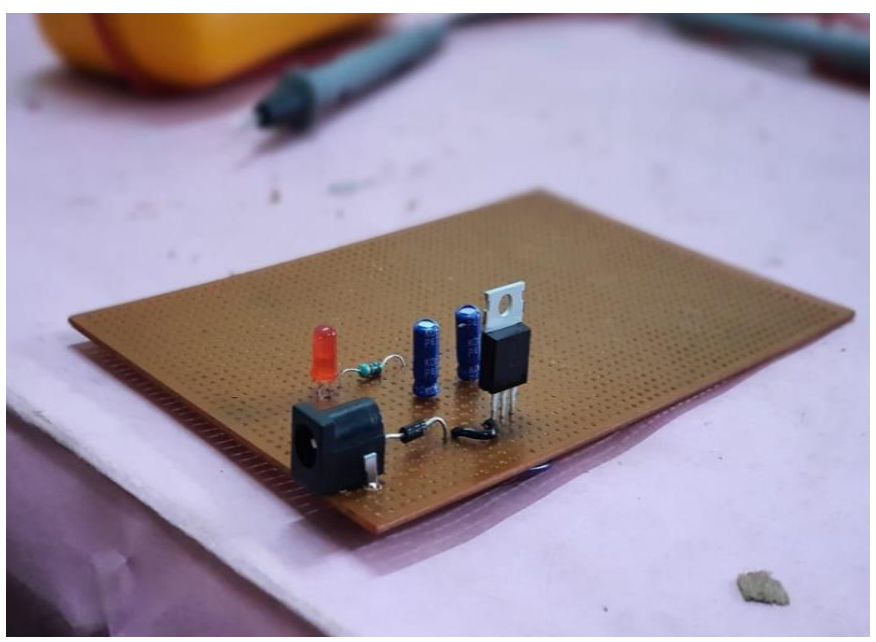

Figure 7- Hardware setup of Power supply

The circuit consists of the battery, diode, capacitors, IC7805 and LED. The 12V supply from the battery which is the input to the power supply circuit is passed through a half diode which ensures unidirectional flow of current. The capacitor $\mathrm{C} 1$ gives the necessary filtering and then the filtered signal is given to the IC7805. The IC7805 is responsible for the voltage conversion. It converts the $12 \mathrm{~V}$ input signal to a $5 \mathrm{~V}$ output signal. The input signal is given to legs 1 and 2 and the output signal is taken from legs 2 and 3, where leg 2 is grounded. Here the voltage difference between the input and output is released in the form of heat. The $5 \mathrm{~V}$ output of the IC7805 is then passed through the capacitor $\mathrm{C} 2$ which provides filtering and also provides transient response. A LED is present which gives the user a visual feedback that the power supply circuit is working. This $5 \mathrm{~V}$ output produced by the power supply circuit is used by both the headlight dimming module and the microcontroller which is part of the engine shutoff module.

\section{2) Headlight Dimming Circuit}

Figure 8 shows the hardware setup of an automatic headlight dimmer circuit using the sensing technique of LDR.

It consists of an LDR, 3 LED to indicate the headlights and a npn transistor $\mathrm{BC} 154$ to drive the light circuit. Here the system automatically senses the opposite vehicle approaching and dims the headlight of the vehicle to a low beam. The prototype shown has a high beam during normal condition and when an opposite high beam approaches the LDR the light intensity of the LEDs reduces to a low beam automatically. The work can reduce the accidents caused in night time due to night temporary blindness which is caused by high beam headlight intensity so that people can drive safely.

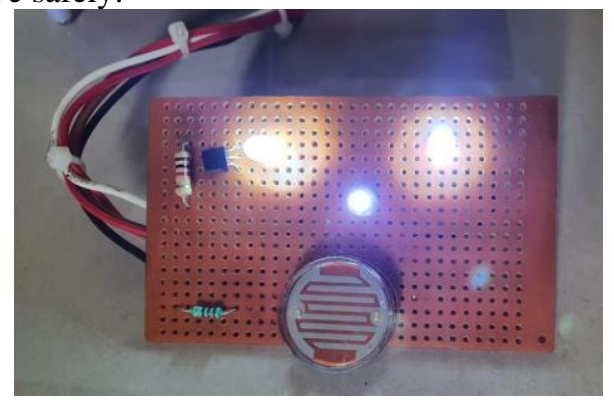

Figure 8- Hardware setup of light dimming circuit.

\section{3) ENGINE ON/OFF CONTROL}

The hardware circuit for engine on/off control is shown in figure. The prototype consists of a microcontroller ESP32, buzzer module, LED indicator lights, a push button switch to show a neutral signal and a $60 \mathrm{rpm}$ DC motor which works with the help of $12 \mathrm{~V}$ relay signal. Also, it consists of two transistors BC154, one to switch the buzzer and one to switch the relay.

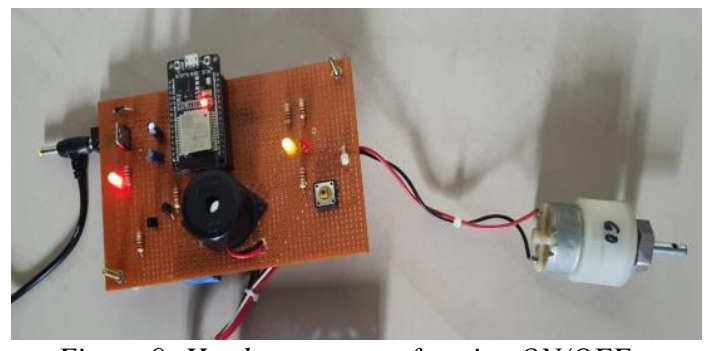

Figure 9- Hardware setup of engine ON/OFF control.

Under normal operation when power is turned on the WiFi device is connected to the server and the engine i.e. the dc motor will turn ON. To show the operation of the neutral signal the push button is used, When the push button is pressed the buzzer starts to alarm. When the push 
button is pressed for more than 10 seconds the engine i.e. the dc motor will turn OFF. This indicates that when the vehicle goes to neutral mode the buzzer alarms for 10 seconds and when the neutral signal persists for more than 10 seconds that means the vehicle is in a traffic signal, then the engine will shut down. When the neutral gear is released, the engine turns $\mathrm{ON}$ automatically.

\section{4) Modular System}

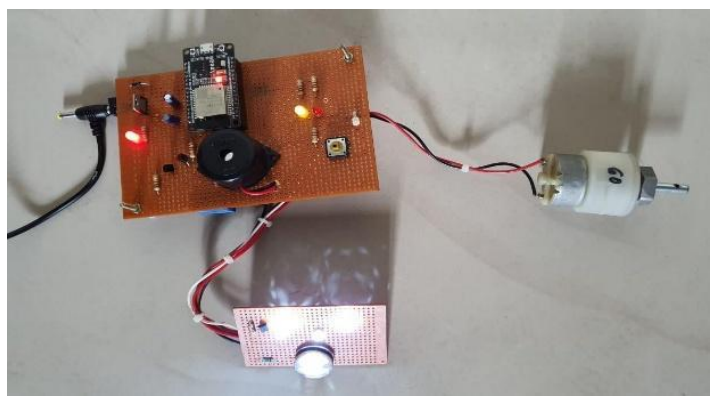

Figure 10-Hardware setup of complete modular system.

The complete modular system of the project is as shown in figure.

\section{Table 2-Vehicle Readings}

$\begin{array}{cc}\text { DATE } & \text { TIME } \\ 2020-06-07 & 22: 46: 59 \\ 2020-06-07 & 23: 18: 39 \\ 2020-06-07 & 23: 19: 08 \\ 2020-06-07 & 23: 19: 14 \\ 2020-06-07 & 23: 19: 20 \\ 2020-06-07 & 23: 19: 26 \\ 2020-06-07 & 23: 19: 33 \\ 2020-06-08 & 13: 59: 56 \\ 2020-06-08 & 14: 00: 40 \\ 2020-06-08 & 14: 00: 47 \\ 2020-06-08 & 14: 00: 53 \\ 2020-06-08 & 14: 00: 59\end{array}$

LDR_VALUE
1
0
0
0
0
0
0
0
48
19
19
25

STATUS
ON
ON
OFF
OFF
OFF
OFF
ON
ON
OFF
OFF
OFF
ON

PACKET

The vehicle readings are shown in table 2 . The details like the turn on and turn off time of the vehicle are shown in the readings. The packet number shows the number of times the device resets. If the readings are continuous the device is turned on. Whenever the device resets the packet number will start counting from "1". Status shows the vehicle status, LDR value shows the light intensity. All the details give the status of the device and vehicle under all conditions.

\section{CONCLUSION}

A serious problem caused by the drivers is the glare during driving which results in accidents during night time. When our eyes are exposed to very bright light it results in glare and here this is due to the bright headlights which results in temporary blindness. This can be avoided when the driver dims the light when an opposite vehicle approaches near. In this project one of the objectives was to avoid such accidents by automatically dimming the headlights.
Another issue faced by the world is pollution due to vehicles. We can see vehicles in traffic signals which are not turned off for minutes which results in environmental pollution. Some extent this pollution can be controlled when the vehicle's engine is shut off when in traffic signals. The second objective of the project was to automatically turn off the engine when the vehicle is in neutral gear.

Combining both the objectives we have designed a modular system incorporating automatic light dimmer and engine on/off control in a single unit. Thus, the implementation of this device in every vehicle in future will not only avoid accidents but also provide a safe and a comfortable driving along with pollution control to some extent.

\section{FUTURE SCOPE}

With the help of this modular system we are able to reduce fuel consumption and thereby reduce pollution caused by motor vehicles. With the help of automatic headlight dimming system, driving becomes much more easy and safe in night time.

This can be further modified in various ways that will be very helpful. By installing a GPS device we will be able to track the location of the vehicle. Using the concept of IoT, more features can be added like live location, tracking, riding modes, real time fuel consumption, remote controlling etc. This can be implemented in future motor vehicles and no doubt it will be of great advantage to people.

\section{REFERENCES}

[1] Okrah. S.K, Williams. E.A Kumassah. F, "Design and Implementation

of Automatic Headlight Dimmer For Vehicles Using Light Dependent Resistor (Ldr) Sensor", International Journal of Emerging Technology and Innovative Engineering Volume 2, Issue 4, April 2016 (ISSN: 2394 - 6598)

[2] Muhammad Usama Qamar, Usman Munawar, Dr.Muhammad Mahmood Aslam Bhutta, Dr. Suhail Aftab Qureshi," Energy \& Cost Efficient Auto Start/Stop Kit System for Motor Bikes: An EcoFriendly Technology", 2017 IEEE Conference on Energy Internet and Energy System Integration (EI2), 04 January 2018.M. King and B. Zhu, "Gaming strategies," in Path Planning to the West, vol. II, S. Tang and M. King, Eds. Xian: Jiaoda Press, 1998, pp. 158-176.

[3] Anand Kishor Asekar," Stop-Start System Using Micro-Hybrid Technology For Increasing Fuel Efficiency", International Journal of Mechanical and Production Engineering, ISSN: 2320-2092, Volume- 1, Issue- 6, Dec-2013.J.-G. Lu, "Title of paper with only the first word capitalized," J. Name Stand. Abbrev., in press.

[4] Pushkar Sevekar, S. B. Dhonde, "Nighttime vehicle detection for intelligent headlight control: A review", 2016 2nd International Conference on Applied and Theoretical Computing and Communication Technology (iCATccT), 21-23 July 2016.M. Young, The Technical Writer's Handbook, Mill Valley, CA: University Science, 1989. 